\title{
Antioxidant Function and Metabolomics Study in Mice after Dietary Supplementation with Methionine
}

\author{
Manrong Yu, ${ }^{1}$ Hui Chen, ${ }^{1}$ Pan Liu, ${ }^{1}$ Mei Yang, ${ }^{1}$ Leqin Zou, ${ }^{1}$ and Dingfu Xiao ${ }^{1,2}$ \\ ${ }^{1}$ College of Animal Science and Technology, Hunan Agricultural University, Changsha Hunan 410128, China \\ ${ }^{2}$ Hunan Collaborative Innovation Center of Animal Production Safety, Changsha Hunan 410128, China \\ Correspondence should be addressed to Dingfu Xiao; xiaodingfu2001@163.com
}

Received 18 February 2020; Accepted 15 May 2020; Published 21 October 2020

Guest Editor: Lei Sun

Copyright (C) 2020 Manrong Yu et al. This is an open access article distributed under the Creative Commons Attribution License, which permits unrestricted use, distribution, and reproduction in any medium, provided the original work is properly cited.

\begin{abstract}
The antioxidant function and metabolic profiles in mice after dietary supplementation with methionine were investigated. The results showed that methionine supplementation enhanced liver GSH-Px activity and upregulated Gpx1 expression in the liver and SOD1 and Gpx4 expressions in the jejunum. Nrf2/Keap1 is involved in oxidative stress, and the western blotting data exhibited that dietary methionine markedly increased Keap1 abundance, while failed to influence the Nrf2 signal. Metabolomics investigation showed that methionine administration increased 2-hydroxypyridine, salicin, and asparagine and reduced $\mathrm{D}$ Talose, maltose, aminoisobutyric acid, and inosine 5'-monophosphate in the liver, which are widely reported to involve in oxidative stress, lipid metabolism, and nucleotides generation. In conclusion, our study provides insights into antioxidant function and liver metabolic profiles in response to dietary supplementation with methionine.
\end{abstract}

\section{Introduction}

Methionine is an essential amino acid and mainly contributes to protein and S-adenosylmethionine (SAM) synthesis in the liver. SAM serves as a versatile methyl donor for nucleic acids and proteins, and the methylation of DNA and proteins is a major regulating mechanism for various physiological functions, including heat shock response $[1,2]$, regulation of intestinal barrier integrity [3], development [4], and gene expression [5]. Meanwhile, methionine has been widely demonstrated to involve in oxidative stress via the transsulfuration pathway for cysteine generation [6]. Cysteine further corresponds for glutathione (GSH) production [6], which is a major thiol group and contributes to antioxidant function against free radical species [7-10].

Here, we found that methionine enhanced antioxidant function via increasing antioxidant enzyme activity and expression. In addition, gas chromatography-mass spectrometry (GC-MS) was performed to investigate the liver metabolic profile after dietary methionine, and the results showed that 7 metabolites (i.e., hydroxypyridine, salicin, asparagine, D-talose, maltose, aminoisobutyric acid, and inosine 5'-monophosphate) were altered and these metabolites are involved in oxidative stress, lipid metabolism, and nucleotides generation.

\section{Methods and Materials}

2.1. Animal and Group. Twenty female ICR mice weighting $22.75 \pm 0.43 \mathrm{~g}$ were assigned into two experimental groups: a control group in which mice received a normal diet (containing $0.43 \%$ methionine) and a Met group in which mice received an additional 0.5\% DL-methionine [11]. Body weight and feed intake were recorded. After three weeks, blood was collected from the eyes, liver, and jejuna, and ilea samples were collected. This study involving animal subjects was approved by the College of Animal Science and Technology, Hunan Agricultural University.

2.2. Antioxidant Function. Serum samples were separated and stored for further analyses. Liver samples were homogenized; then, the supernatant was collected for further analyses. Antioxidant indexes (i.e., superoxide dismutase (SOD), 
TABLE 1: PCR primer sequences: the forward primers (F) and the reverse primers (R) used in this study.

\begin{tabular}{|c|c|c|c|}
\hline Gene & Accession no. & Nucleotide sequence of primers $\left(5^{\prime}-3^{\prime}\right)$ & Size (bp) \\
\hline$\beta$-Actin & NM_007393.3 & $\begin{array}{l}\text { F:GTCCACCTTCCAGCAGATGT } \\
\text { R:GAAAGGGTGTAAAACGCAGC }\end{array}$ & 117 \\
\hline CAT & XM_006498624.1 & $\begin{array}{l}\text { F:AATATCGTGGGTGACCTCAA } \\
\text { R:CAGATGAAGCAGTGGAAGGA }\end{array}$ & 243 \\
\hline $\mathrm{ZnCuSOD}$ & NM_011434.1 & $\begin{array}{l}\text { F:CCACTGCAGGACCTCATTTT } \\
\text { R:CACCTTTGCCCAAGTCATCT }\end{array}$ & 216 \\
\hline Gpx1 & NM_008160.6 & $\begin{array}{l}\text { F:GGTTCGAGCCCAATTTTACA } \\
\text { R:CCCACCAGGAACTTCTCAAA }\end{array}$ & 199 \\
\hline Gpx4 & NM_001037741.3 & $\begin{array}{l}\text { F:CTCCATGCACGAATTCTCAG } \\
\text { R:ACGTCAGTTTTGCCTCATTG }\end{array}$ & 117 \\
\hline $\mathrm{UCP} 2$ & NM_011671.5 & $\begin{array}{c}\text { F:TAGTGCGCACCGCAGCC } \\
\text { R:AGCTCATCTGGCGCTGCAG }\end{array}$ & 126 \\
\hline
\end{tabular}

TABLE 2: Growth performance after dietary supplementation with methionine. IBD: initial body weight; FBW: final body weight; AFI: average feed intake; ABWG: average body weight gain; F : G: the ratio of feed intake to weight gain.

\begin{tabular}{lccccc}
\hline Item & IBW & FBW & AFI & ABWG & F:G \\
\hline Cont & $22.26 \pm 0.37$ & $28.27 \pm 0.53$ & $5.65 \pm 0.05$ & $0.30 \pm 0.01$ & $19.36 \pm 0.88$ \\
Met & $23.24 \pm 0.50$ & $26.84 \pm 0.47$ & $5.76 \pm 0.02$ & $0.27 \pm 0.02$ & $21.85 \pm 1.26$ \\
\hline
\end{tabular}

glutathione peroxidase (GSH-Px), and catalase (CAT)) were tested (Nanjing Jiancheng, China) [12].

2.3. RT-PCR. RNA isolation and cDNA synthesis were conducted according to previous studies [13]. Primer 5.0 was used to design the primer (Table 1). RT-PCR was performed according to previous studies [14-18].

2.4. Western Blot. Liver proteins were extracted by the Thermo Fisher kits (Waltham, MA, USA) and separated by SDS-PAGE electrophoresis $[14,19,20]$. Then, the separated proteins were transferred onto PVDF membranes (Millipore, MA, USA) for the incubation of antibodies, including taste receptor type 1 member 1 (T1R1), taste receptor type 1 member 3 (T1R3), Kelch-like ECH-associating protein 1 (Keap1), nuclear factor erythroid 2-related factor 2 (Nrf2), and uncoupling protein 2 (Ucp2) (Abcam Bio).

2.5. Gas Chromatography-Mass Spectrometry (GC-MS) Analysis. Metabolomics study of liver samples was investigated (Agilent 7890A, Agilent, USA), and data were analyzed by the Chroma TOF 4.3X software (LECO Corporation, USA) and LECO-Fiehn Rtx5 database [21-25].

2.6. Statistical Analysis. All data were analyzed using the Students' $T$ test (SPSS 17.0 software). Data are expressed as the mean \pm sem. Values in the same row with different superscripts are significant $(P<0.05)$.

\section{Results}

3.1. Growth Performance. Dietary $0.5 \%$ methionine had no effect on the body weight gain, average feed intake, and the ratio of feed intake to body weight gain $(P<0.05)$ (Table 2$)$.
TABLE 3: GSH-Px, SOD, and CAT activities in the serum and liver after dietary supplementation with methionine. The * means the difference is significant between the two groups $(P<0.05)$.

\begin{tabular}{lccc}
\hline Item & GSH-Px & SOD & CAT \\
\hline $\begin{array}{l}\text { Serum } \\
\text { (U/ml) }\end{array}$ & & & \\
$\quad$ Cont & $17.89 \pm 1.22$ & $116.74 \pm 20.48$ & $366.52 \pm 135.33$ \\
$\quad$ Met & $28.95 \pm 7.78$ & $131.06 \pm 12.51$ & $216.77 \pm 34.27$ \\
Liver & & & \\
(U/mgprot) & & & \\
$\quad$ Cont & $26.80 \pm 2.35$ & $2342.63 \pm 50.94$ & $188.12 \pm 15.24$ \\
$\quad$ Met & $152.88 \pm 30.01^{*}$ & $2398.21 \pm 70.69$ & $116.08 \pm 20.47^{*}$ \\
\hline
\end{tabular}

3.2. Antioxidant Function. Serum and liver GSH-Px, SOD, and CAT were measured to evaluate the antioxidant function after dietary supplementation with methionine (Table 3 ). In the serum, we did not find any significant difference on GSH-Px, SOD, and CAT activities $(P>0.05)$. In addition, methionine supplementation markedly enhanced liver GSHPx activity $(P<0.05)$, while liver CAT activity was inhibited in the Met group $(P<0.05)$.

We further determined the expression of antioxidant genes (CAT, SOD1, Gpx, Gpx4, and UCP2) in the liver, jejunum, and ileum (Figure 1). In the liver, methionine markedly upregulated the Gpxl expression $(P<0.05)$. In the jejunum, methionine markedly increased SOD1 and Gpx4 mRNA abundances $(P<0.05)$.

3.3. T1R1 and T1R3. Intestinal T1R1 and T1R 3 were determined via western blot (Figure 2). In the jejunum and ileum, 

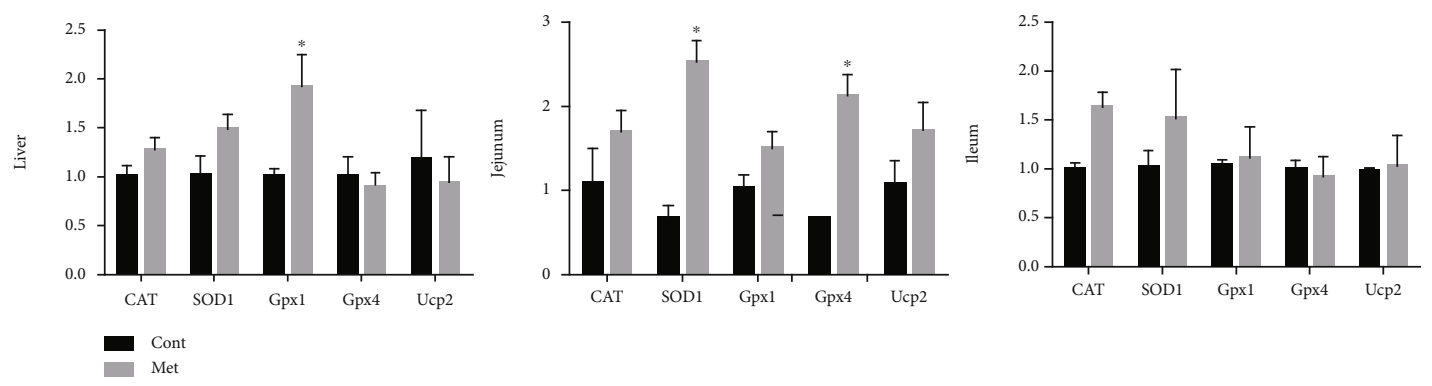

FIGURE 1: The expression of antioxidant genes (CAT, SOD1, Gpx, Gpx4, and UCP2) in the liver, jejunum, and ileum.

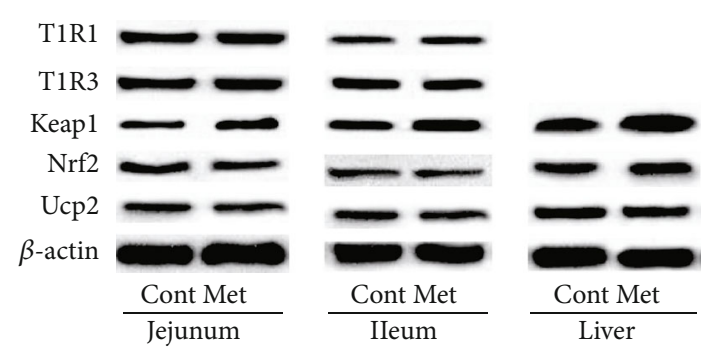

FIGURE 2: Western blot: T1R1 and T1R3 of jejunum, ileum, and liver.

dietary supplementation failed to influence T1R1 and T1R3 abundance $(P>0.05)$.

3.4. Nrf2/Keap1 Signal. In this study, dietary supplementation significantly enhanced the liver and ileal Keap1 expression $(P<0.05)$, but Nrf2 expression was not changed $(P>0.05)$.

3.5. Metabolic Profiles Analyzed by GC-MS. Metabolites in the liver were analyzed from 534 peaks (Figure 3(a)). PLSDA score plots exhibited that dietary supplementation with methionine leaded distinctive metabolic profiles in mice compared with the control mice (Figure 3(b) and 3(c)).

The identified potential markers after dietary supplementation with methionine are listed in Table 4. Dietary methionine significantly increased liver 2-hydroxypyridine, salicin, and asparagine concentrations. Meanwhile, liver D-talose, maltose, aminoisobutyric acid, and inosine 5'monophosphate were markedly reduced after dietary methionine.

\section{Discussion}

Methionine is a limiting AA in most cereal soybean-based diets for growing pigs [26]. Recently, methionine has been reported to involve in lipid metabolism [27], translational capacity [28], autophagy [29], and maintenance and differentiation of pluripotent stem cells [30]. Meanwhile, dietary methionine or methionine restriction has been demonstrated to influence growing performance $[31,32]$, while the current study did not observed any significant difference in the growth performance in mice. In addition, we found that dietary supplementation with methionine enhanced antioxidant function and upregulated liver Keap1 expression. Mean- while, 7 metabolites were found related to dietary methionine based on GC-MS-based metabolomics investigation.

Methionine can sustain cellular redox homeostasis via the generation of cysteine, which is a substance for GSH synthesis $[6,33]$. In this study, we found that dietary methionine increased antioxidant function evidenced by the enhanced serum GSH-Px activity and upregulation of Gpx1, Gpx4, and SOD1. Methionine can form cysteine via the transsulfuration pathway to serve as a precursor for GSH, hydrogen sulfide, and taurine [6]. These metabolites have been demonstrated to mediate oxidative stress via scavenging hydroxyl radical and superoxide directly and serving as a cofactor for the antioxidant enzymes, including GSH peroxidase (Gpx) [6]. Meanwhile, Campbell et al. reported that methionine affects the oxidative branch of the pentose phosphate pathway and increased abundance of the NADPH producing enzyme 6PGDH, which further plays a protective role against oxidative stress [34].

Nrf2/keap1 signaling pathway is widely involved in oxidative stress and regulates the expression of antioxidant genes $[12,14,15]$. Normally, Nrf2 protein is sequestered in the cytosol via targeting Keap1 and soon be degraded under cellular homeostasis [35]. Although Keap1 was upregulated after dietary methionine, liver, jejunal, and ileal Nrf2 expression was not changed in this study. Nrf2 activation was observed under methionine restriction $[36,37]$, which further regulated antioxidant genes and oxidative stress.

Metabolomics investigation revealed 7 metabolites after dietary methionine, including hydroxypyridine, salicin, asparagine, D-Talose, maltose, aminoisobutyric acid, and inosine 5'-monophosphate. Salicin has been studied as a potent antiinflammatory agent and exhibits an anti-inflammatory and antioxidant functions in various inflammation-related diseases [38, 39]. Asparagine is a metabolic product of the aspartate, which has been demonstrated to alleviate diquat-induced oxidative injury in piglets [40]. Thus, the increased contents of salicin and asparagine in the Met group may indicate methionine mediates inflammation and oxidative stress via indirectly affecting salicin and asparagine concentrations. Aminoisobutyric acid, a low molecular myokine, contributes to the conversion of white adipose tissue into brown fat, accelerating the breakdown of lipids into heat, water, and $\mathrm{CO}_{2}$ [41]. Previous reports also demonstrated that methionine regulates lipid metabolism via increasing metabolic flexibility and overall insulin sensitivity [42]. Inosine $5^{\prime}$-monophosphate is an intermediate of purine metabolism and serves as a 


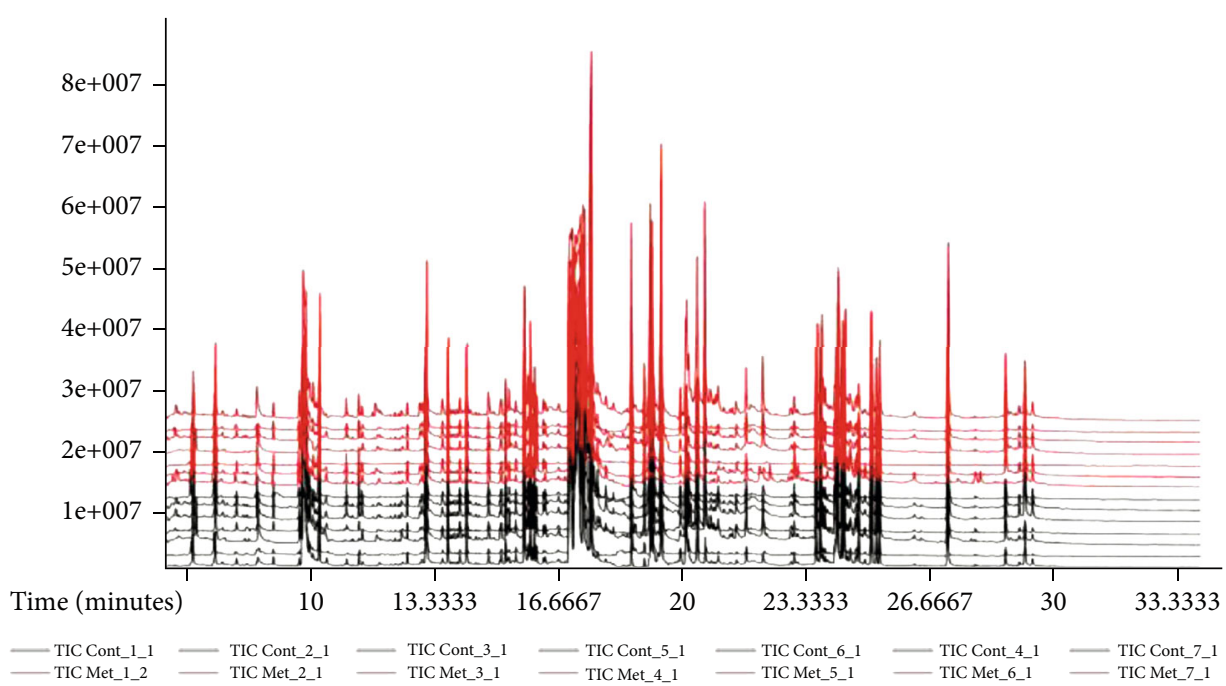

(a)

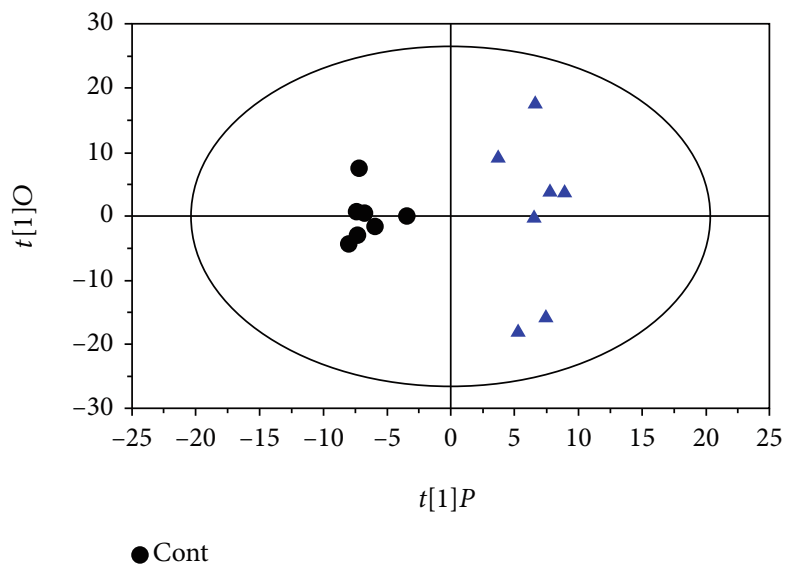

Met

(b)

SIMCA.M5 (OPLS-DA): validate model

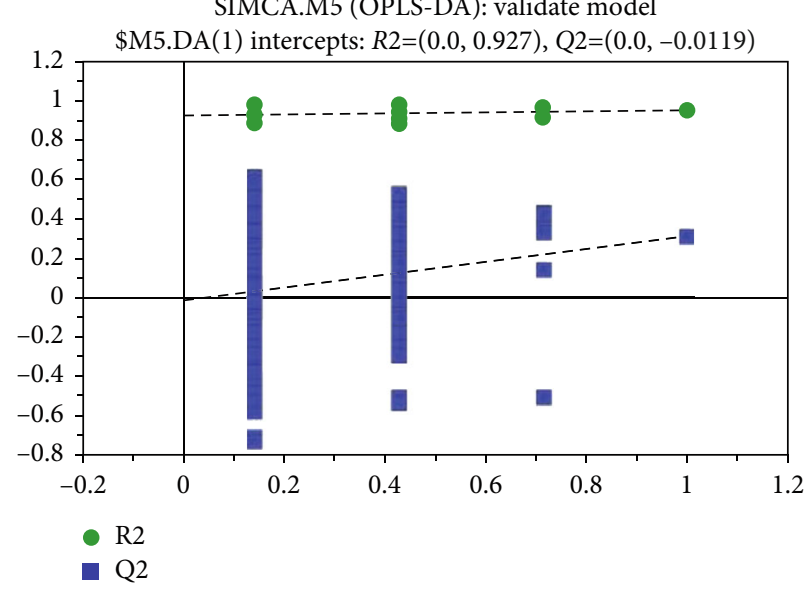

(c)

Figure 3: Metabolites identified from 534 peaks in the chromatograms and PLS-DA score plots.

TABLE 4: List of differential metabolites between the control and Met groups.

\begin{tabular}{lccccccccc}
\hline ID & Peak & R.T. & Count & Mass & Cont & Met & VIP & $P$ value & Fold change \\
\hline 13 & 2-hydroxypyridine & 6.61 & 29 & 152 & 0.64 & 1.32 & 1.94 & 0.03 & 2.05 \\
421 & D-Talose & 17.38 & 16 & 235 & 0.25 & 0.00 & 1.92 & 0.04 & 0.00 \\
662 & Maltose & 24.22 & 17 & 361 & 4.39 & 1.10 & 1.96 & 0.03 & 0.25 \\
204 & Aminoisobutyric acid & 12.31 & 23 & 174 & 0.11 & 0.04 & 2.04 & 0.04 & 0.36 \\
597 & Salicin & 22.42 & 29 & 169 & 0.02 & 0.03 & 1.99 & 0.04 & 2.08 \\
711 & Inosine 5'-monophosphate & 25.87 & 19 & 169 & 0.01 & 0.00 & 1.94 & $<0.01$ & 0.09 \\
300 & Asparagine & 14.37 & 19 & 229 & 0.00 & 0.01 & 2.69 & $<0.01$ & 3980383.06 \\
\hline
\end{tabular}

precursor for other nucleotides used for metabolic functions $[43,44]$. Thus, we speculated that dietary methionine serves as a regulator in nucleotide metabolism, which was further demonstrated by Benjamin P. Tu who reported that methionine regulates translational capacity through modulation of tRNA thiolation [28].
In conclusion, dietary supplementation with methionine enhanced antioxidant function. Meanwhile, metabolomics investigation revealed that methionine influenced 7 metabolites, including hydroxypyridine, salicin, asparagine, D-Talose, maltose, aminoisobutyric acid, and inosine 5'monophosphate. 


\section{Data Availability}

The data used to support the findings of this study are available from the corresponding author upon request.

\section{Conflicts of Interest}

The authors have declared no conflict of interest.

\section{Authors' Contributions}

D.F., Xiao designed the experiment. M.R Yu, D.F. Xiao, H. Chen, P. Liu, L.Q. Zou, and M. Yang conducted the experiment. H. Chen performed the data analysis. D.F. Xiao wrote the manuscript.

\section{Acknowledgments}

This study was jointly supported by the National Natural Science Foundation of China (No.31872991) and the "double first Class" Construction Project of Hunan Agricultural University (kxk201801004).

\section{References}

[1] J. Zhou, J. Wan, X. Gao, X. Zhang, S. R. Jaffrey, and S. B. Qian, "Dynamic m(6)A mRNA methylation directs translational control of heat shock response," Nature, vol. 526, no. 7574, pp. 591-594, 2015.

[2] A. Bhattacharya, M. Sharma, C. Packianathan, B. P. Rosen, P. Leprohon, and M. Ouellette, "Genomewide Analysis of Mode of Action of the S-Adenosylmethionine Analogue Sinefungin in Leishmania infantum," Msystems, vol. 4, p. e00416, 2019.

[3] P. G. Thomes, N. A. Osna, S. M. Bligh, D. J. Tuma, and K. K. Kharbanda, "Role of defective methylation reactions in ethanol-induced dysregulation of intestinal barrier integrity," Biochemical Pharmacology, vol. 96, no. 1, pp. 30-38, 2015.

[4] C. Li, S. Guo, M. Zhang, J. Gao, and Y. Guo, "DNA methylation and histone modification patterns during the late embryonic and early postnatal development of chickens," Poultry Science, vol. 94, no. 4, pp. 706-721, 2015.

[5] J. Dunn, H. Qiu, S. Kim et al., "Flow-dependent epigenetic DNA methylation regulates endothelial gene expression and atherosclerosis," The Journal of Clinical Investigation, vol. 124, no. 7, pp. 3187-3199, 2014.

[6] J. Yin, W. Ren, G. Yang et al., "1-Cysteine metabolism and its nutritional implications," Molecular Nutrition \& Food Research, vol. 60, no. 1, pp. 134-146, 2016.

[7] H. A. El Hendy and H. A. Al-Gemeai, "Effect of broccoli intake on antioxidant in the liver and kidney tissues of hyperglycemic rats," Integrative food, nutrition and metabolism, vol. 1, pp. 83-86, 2014.

[8] M. J. McCann, J. E. Dalziel, R. Bibiloni, and M. P. G. Barnett, "An integrated approach to assessing the bio-activity of nutrients in vitro: the anti-oxidant effects of catechin and chlorogenic acid as an example," Integrative food, nutrition and metabolism, vol. 2, pp. 197-204, 2015.

[9] A. B. Shori and A. S. Baba, "Fermented milk derives bioactive peptides with antihypertensive effects," Integrative food, nutrition and metabolism, vol. 2, pp. 178-181, 2015.
[10] S. Mileva, B. Galunska, M. Gospodinova, D. Gerova, and D. Svinarov, "Vitamin D3 status in children with acute diarrhea," Integrative food, nutrition and metabolism, vol. 1, pp. 1-6, 2014.

[11] W. Ren, S. Chen, J. Yin et al., "Dietary arginine supplementation of mice alters the microbial population and activates intestinal innate immunity," The Journal of Nutrition, vol. 144, no. 6, pp. 988-995, 2014.

[12] J. Yin, J. Duan, Z. Cui, W. Ren, T. Li, and Y. Yin, "Hydrogen peroxide-induced oxidative stress activates NF- $\kappa \mathrm{B}$ and Nrf2/Keap1 signals and triggers autophagy in piglets," $R S C$ Advances, vol. 5, no. 20, pp. 15479-15486, 2015.

[13] D. Xiao, Z. Tang, Y. Yin et al., "Effects of dietary administering chitosan on growth performance, jejunal morphology, jejunal mucosal sIgA, occludin, claudin-1 and TLR4 expression in weaned piglets challenged by enterotoxigenic Escherichia coli," International Immunopharmacology, vol. 17, no. 3, pp. 670-676, 2013.

[14] J. Yin, W. Ren, G. Liu et al., "Birth oxidative stress and the development of an antioxidant system in newborn piglets," Free Radical Research, vol. 47, no. 12, pp. 1027-1035, 2013.

[15] J. Yin, M. M. Wu, H. Xiao et al., "Development of an antioxidant system after early weaning in piglets," Journal of Animal Science, vol. 92, no. 2, pp. 612-619, 2014.

[16] J. Yin, Y. Li, H. Han et al., "Melatonin reprogramming of gut microbiota improves lipid dysmetabolism in high-fat diet-fed mice," Journal of Pineal Research, vol. 65, no. 4, article e12524, 2018.

[17] Y. Duan, Y. Zhong, H. Xiao et al., "Gut microbiota mediates the protective effects of dietary $\beta$-hydroxy- $\beta$-methylbutyrate (HMB) against obesity induced by high-fat diets," The FASEB Journal, vol. 33, no. 9, pp. 10019-10033, 2019.

[18] I. Chouikha, D. E. Sturdevant, C. Jarrett, Y. C. Sun, and B. J. Hinnebusch, "Differential Gene Expression Patterns ofYersinia pestisandYersinia pseudotuberculosisduring Infection and Biofilm Formation in the Flea Digestive Tract," Msystems, vol. 4, no. 1, 2019.

[19] J. Yin, Y. Li, H. Han et al., "Effects of lysine deficiency and Lys-Lys dipeptide on cellular apoptosis and amino acids metabolism," Molecular Nutrition \& Food Research, vol. 61, no. 9, 2017.

[20] J. Yin, Y. Li, X. Zhu et al., "Effects of long-term protein restriction on meat quality, muscle amino acids, and amino acid transporters in pigs," Journal of Agricultural and Food Chemistry, vol. 65, no. 42, pp. 9297-9304, 2017.

[21] J. Yin, F. Li, X. Kong et al., "Dietary xylo-oligosaccharide improves intestinal functions in weaned piglets," Food \& Function, vol. 10, no. 5, pp. 2701-2709, 2019.

[22] J. Yin, Y. Li, H. Han et al., "Long-term effects of lysine concentration on growth performance, intestinal microbiome, and metabolic profiles in a pig model," Food \& Function, vol. 9, no. 8, pp. 4153-4163, 2018.

[23] M. L. Han, Y. Zhu, D. J. Creek et al., "Comparative metabolomics and transcriptomics reveal multiple pathways associated with polymyxin killing in Pseudomonas aeruginosa," Msystems, vol. 4, no. 1, 2019.

[24] M. A. Henson, G. Orazi, P. Phalak, and G. A. O'Toole, "Metabolic Modeling of Cystic Fibrosis Airway Communities Predicts Mechanisms of Pathogen Dominance," Msystems, vol. 4, 2019.

[25] Y. Zhu, J. X. Zhao, M. H. M. Maifiah, T. Velkov, F. Schreiber, and J. Li, "Metabolic responses to polymyxin treatment in 
Acinetobacter baumannii ATCC 19606: integrating transcriptomics and metabolomics with genome-scale metabolic modeling," Msystems, vol. 4, no. 1, 2019.

[26] S. Metayer, I. Seiliez, A. Collin et al., "Mechanisms through which sulfur amino acids control protein metabolism and oxidative status," The Journal of Nutritional Biochemistry, vol. 19, no. 4, pp. 207-215, 2008.

[27] P. Jha, A. Knopf, H. Koefeler et al., "Role of adipose tissue in methionine-choline-deficient model of non-alcoholic steatohepatitis (NASH)," Biochimica et Biophysica Acta, vol. 1842, no. 7, pp. 959-970, 2014.

[28] S. Laxman, B. M. Sutter, X. Wu et al., "Sulfur amino acids regulate translational capacity and metabolic homeostasis through modulation of tRNA thiolation," Cell, vol. 154, no. 2, pp. 416-429, 2013.

[29] B. M. Sutter, X. Wu, S. Laxman, and B. P. Tu, "Methionine inhibits autophagy and promotes growth by inducing the SAM-responsive methylation of PP2A," Cell, vol. 154, no. 2, pp. 403-415, 2013.

[30] N. Shiraki, Y. Shiraki, T. Tsuyama et al., "Methionine metabolism regulates maintenance and differentiation of human pluripotent stem cells," Cell Metabolism, vol. 19, no. 5, pp. 780$794,2014$.

[31] F. Hirai and T. Matsui, "Status of food intake and elemental nutrition in patients with Crohn's disease," Integrative food, nutrition and metabolism, vol. 2, pp. 148-150, 2015.

[32] J. Eastep and G. Chen, "The relationships of high-fat diet and metabolism of lipophilic vitamins," Integrative food, nutrition and metabolism, vol. 2, pp. 174-179, 2015.

[33] S. Eriksson, J. R. Prigge, E. A. Talago, E. S. J. Arner, and E. E. Schmidt, "Dietary methionine can sustain cytosolic redox homeostasis in the mouse liver," Nature communications, vol. $6,2015$.

[34] K. Campbell, J. Vowinckel, M. A. Keller, and M. Ralser, "Methionine metabolism alters oxidative stress resistance via the pentose phosphate pathway," Antioxidants \& Redox Signaling, vol. 24, no. 10, pp. 543-547, 2016.

[35] J. Yin, W. K. Ren, X. S. Wu et al., "Oxidative stress-mediated signaling pathways: a review," Journal of Food, Agriculture and Environment, vol. 11, pp. 132-139, 2013.

[36] Y. K. Zhang, R. L. Yeager, Y. Tanaka, and C. D. Klaassen, "Enhanced expression of Nrf2 in mice attenuates the fatty liver produced by a methionine- and choline-deficient diet," Toxicology and Applied Pharmacology, vol. 245, no. 3, pp. 326$334,2010$.

[37] A. H. Lin, H. W. Chen, C. T. Liu, C. W. Tsai, and C. K. Lii, "Activation of Nrf2 Is Required for Up-Regulation of the $\pi$ Class of GlutathioneS-Transferase in Rat Primary Hepatocytes withl-MethionineStarvation," Journal of agricultural and food chemistry, vol. 60, no. 26, pp. 6537-6545, 2012.

[38] Y. Li, Q. Wu, Y. Deng et al., "D(-)-Salicin inhibits the LPSinduced inflammation in RAW264.7 cells and mouse models," International immunopharmacology, vol. 26, no. 2, pp. 286294, 2015.

[39] N. Verma, R. Verma, R. Kumari, R. Ranjha, and J. Paul, "Effect of salicin on gut inflammation and on selected groups of gut microbiota in dextran sodium sulfate induced mouse model of colitis," Inflammation Research, vol. 63, no. 2, pp. 161169, 2014.

[40] J. Yin, M. Liu, W. Ren et al., "Effects of dietary supplementation with glutamate and aspartate on diquat-induced oxi- dative stress in piglets," PLoS One, vol. 10, no. 4, article e0122893, 2015.

[41] E. Ginter and V. Simko, "Recent data on obesity research: $\beta$ aminoisobutyric acid," Bratislavske lekarske listy, vol. 115, no. 8, pp. 492-493, 2014.

[42] M. L. Orgeron, K. P. Stone, D. Wanders, C. C. Cortez, N. T. Van, and T. W. Gettys, "The Impact of Dietary Methionine Restriction on Biomarkers of Metabolic Health," Progress in molecular biology and translational science, vol. 121, pp. 351376, 2014.

[43] H. H. Stein and D. Y. Kil, "Reduced use of antibiotic growth promoters in diets fed to weanling pigs: dietary tools, part 2," Animal Biotechnology, vol. 17, no. 2, pp. 217-231, 2006.

[44] T. Ma, L. Xu, H. Wang et al., "Mining the key regulatory genes of chicken inosine 5'-monophosphate metabolism based on time series microarray data," Journal of Animal Science and Biotechnology, vol. 6, no. 1, p. 21, 2015. 